Military Technical College Kobry El-Kobbah, Cairo, Egypt

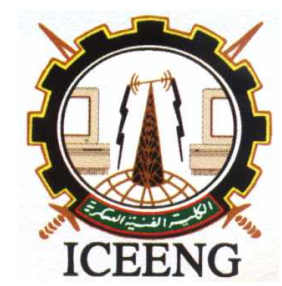

$6^{\text {th }}$ International Conference on Electrical Engineering ICEENG 2008

\title{
PERFORMANCE ANALYSIS OF PARTIAL BAND JAMMED HYBRID SFH/DS SSMA OVER FADING CHANNEL
}

\author{
By
}

Abd Elmoneim M. Fouda * $\quad$ Ezz Eldin Farouk** Bassem Ibrahim Sheta **

\section{$\underline{\text { Abstract: }}$}

A hybrid slow frequency hopping/direct sequence spread spectrum multiple access (hybrid SFH/DS SSMA) are attractive because they can combine the advantages of the pure SH and the pure DS, while avoiding some of their disadvantages. The paper presents the BER performance analysis of the hybrid SFH/DS SSMA operating through fading channel. Moreover, such hybrid system is subjected to the effect of partial band noise or jamming. The performance is discussed assuming noncoherent hybrid SFH/DS system, which employs independent random direct sequences and random memoryless frequency hopping patterns. The system is operating through Rician and Rayleigh fading channel, and the system is employing frequency shift keying FSK modulation technique. A closed form expression for the hybrid system BER is derived for the system under consideration. The performance measures including BER against signal to noise ratio SNR, and jamming to signal ratio JSR are presented and plotted for the system under consideration.

\footnotetext{
* Modern Academy

** Egyptian Armed Forces
} 


\section{I-INTRODUCTION}

The processing gain of the SS system is the ratio of the bandwidth of a spread spectrum signal to the bandwidth of the data signal. The DS processing gain is the ratio $T / T_{c}$ which is equal to $N$ that represents the number of chips within the data bit duration. Where $T$ is the bit duration, and $T_{c}$ is the chip duration. While the processing gain of the FH system is the number of frequency hops q.

We utilize the feature of the increased processing gain of the hybrid SFH/DS SS, that it outperforms both the pure FH SSMA and the pure DS SSMA. The processing gain of a hybrid FH/ DS SS systems can be expressed as [1]:

$\mathrm{G}_{\mathrm{HYP}}=\mathrm{G}_{\mathrm{DS}} \cdot \mathrm{G}_{\mathrm{FH}}=\mathrm{N} \cdot \mathrm{q}$

where $G_{D S}$ is the

DS-SS processing gain, and $\mathrm{G}_{\mathrm{FH}}$ is the FH-SS processing gain .

the paper is organized as follows, in part II we introduce to the system model describing the channel model, the transmitted and received signal format of such a hybrid system, as well as the jamming model. While in section III we develop to drive the formula of the BER as performance measure. In section IV we give the different numerical results. Finally a conclusion is adduced in section V .

\section{II-SYSTEM MODEL}

\section{A- Channel model}

The channel under consideration can be represented as the link between the $\mathrm{k}^{\text {th }}$ user and the base station, it is characterized by a lowpass equivalent transfer function or the channel impulse response given by [2]:

$\mathrm{h}_{\mathrm{k}}(\mathrm{t})=\beta_{\mathrm{kl}} \delta\left(\mathrm{t}-\tau_{\mathrm{kl}}\right) \exp \left(\mathrm{j} \gamma_{\mathrm{kl}}\right)$

where all the random variable $\beta, \tau$, and $\gamma$ are assumed to be independent for different values of $\mathrm{k}$ and $l$ . $\mathrm{k}$ is referring to the user number, while $l$ is referring to the path number. $\tau_{\mathrm{kl}}$ is the delays which are assumed to be uniformly distributed random variables over the one tone symbol duration, while the delay belongs to $\left[0, T / \mathrm{N}_{\mathrm{t}}\right]$ in the case of $\mathrm{N}_{\mathrm{t}}$ multi tones. $\beta_{\mathrm{kl}}$ is the path gain.

Assuming that there are a total number of $\mathrm{K}$ user competing for transmission over the prescribed channel. The model of the $\mathrm{k}^{\text {th }}$ transmitted signal for the system under consideration is [3]:

$S_{k}(t)=\sqrt{2 P} b_{k}(t) a_{k}(t) \cos \left\{2 \pi\left(f_{c}+f_{h k}(t)\right) t+\theta_{k}(t)+\alpha_{k}(t)\right\}$

Where $\mathrm{p}$ is the signal power, $b_{k}(t)$ is a random binary data sequence of which pulse duration is $T_{b}$ seconds and $a_{k}(t)$ is a spreading sequence with period $N$. the chip duration is $T_{c}=T_{b} / N$ seconds. The DS spread signal changes its carrier frequencies $f_{c}$ to be $f_{c}+f_{h k}(t)$, and $f_{h k}(t)$ is the 
frequency hopping pattern, it is selected from the sequence of $j$ frequencies.$\{j=1,2, \ldots . q) \cdot q$ is the number of frequencies available for hopping. And $\alpha_{k}(t)$ is the phase waveform of the $\mathrm{k}^{\text {th }}$ frequency hopper.

A receiver model is shown in Fig. 1. The first user's signal is desired and the perfect time synchronization is assumed in the receiver. First of all, we consider the case of the $\mathrm{k}^{\text {th }}$ user's signal corrupted by the other K-1 interfering users and the single tone jamming signal. The received signal is given by:

$\mathrm{r}(\mathrm{t})=\Sigma \mathrm{S}_{\mathrm{k}}\left(\mathrm{t}-\tau_{\mathrm{k}}\right)+\mathrm{J}(\mathrm{t})+\mathrm{n}(\mathrm{t})$

$=\sqrt{2 P} b_{k}\left(t-\tau_{k}\right) a_{k}\left(t-\tau_{k}\right) \cos \left\{2 \pi\left(f_{c}+f_{h k}\left(t-\tau_{k}\right)\right) t+\theta_{k}\left(t-\tau_{k}\right)+\alpha_{k}\left(t-\tau_{k}\right)\right\}+J(t)+n(t)$

\section{B- jamming model}

A wideband jammer emits a noise-like signal with power $\mathrm{J}$ distributed uniformly across the $\mathrm{B} \mathrm{Hz}$ bandwidth, typically the jammer bandwidth $>$ the signal bandwidth i.e., B $>B_{s}$. Using jammer power $\mathrm{J}$, the jammer density $\mathrm{J}_{\mathrm{o}}=\mathrm{J} / \mathrm{B}$ measured in Watt $/ \mathrm{Hz}$. The target is attempting to receive a signal with bit energy $E_{b}$, . The signal-to-jammer ratio (SJR) is defined as $\gamma_{j}=E_{b} / J_{0}$.

The wideband jamming is not necessarily the optimum jammer strategy. Another type called a partialband jammer $(\mathrm{PBJ})$ that concentrates its power in a $\mathrm{\rho B} \mathrm{Hz}$ subband of the communications band, where $\rho$ is called the fractional jammer bandwidth. The value of $\rho$ is limited to $0<\rho<1$, the signal will jam with probability $\rho$. When the signal is jammed, effective jammer density is $J_{0} / \rho$. The overall error probability in the case of PBJ is approximated by [4] :

$\mathrm{P}_{\mathrm{ePBJ}}=0.5 \rho \exp \left(-0.5 \rho \gamma_{\mathrm{j}}\right)$

It is shown in Fig.2 the partial band jammer BER as a function of signal to jammer ratio, given that different values of fractional jammer bandwidth it is noted from the plot that for smaller values of SJR (about $4 \mathrm{~dB}$ ), the more the fractional jammer bandwidth the increase of the partial band jammer BER , while for larger value of SJR the more the fractional jammer bandwidth the decrease of the partial band jammer BER.

\section{III- BER ANALYSIS}

Now we develop to investigate the performance of a hybrid SFH/DS employing frequency shift keying modulation, and with noncoherent reception, assuming that the communication link in the presence of partial band jamming. 
To calculate such BER in the case of hybrid system suffered from Rician fading and under the effect of PBJ. We denote. $\gamma_{0}$ is the average desired signal to the sum of the multipath interference, multiple access interference and the channel noise ratio. It can be expressed for the system with no jamming, and employing FSK modulation as [5]:

$$
\gamma_{0}=\left[\left(\mathrm{N}_{\mathrm{o}} / 2 \mathrm{E}_{\mathrm{b}}\right)+\left(\mathrm{K}_{\mathrm{f}} \mathrm{L} / 3 \mathrm{~N}\right)\right]^{-1}=\left[(2 . \mathrm{SNR})^{-1}+\left(\mathrm{K}_{\mathrm{f}} \mathrm{L} / 3 \mathrm{~N}\right)\right]^{-1}
$$

On the other hand, when the e system is subjected to partial band jamming effect, let $\gamma_{1}$ is the average desired signal to the sum of the multipart interference, multiple access interference, the channel noise, and partial band noise ratio. it can be expressed $\gamma_{1}$ and employing FSK modulation as then $\mathrm{N}_{0}$ is replaced by, $\mathrm{N}_{0}+\rho^{-1} \mathrm{~N}_{\mathrm{j}}$ where $\rho$ represent the jamming fraction ratio [6], then we express :

$\gamma_{1}=\left[\left(\mathrm{N}_{\mathrm{o}}+\rho^{-1} \mathrm{~N}_{\mathrm{j}}\right) / 2 \mathrm{E}_{\mathrm{b}}+\left(\mathrm{K}_{\mathrm{f}} \mathrm{L} / 3 \mathrm{~N}\right)\right]^{-1}=\left[(2 \cdot \mathrm{SNR})^{-1}+(2 \cdot \rho \cdot \mathrm{SJR})^{-1}+\left(\mathrm{K}_{\mathrm{f}} \mathrm{L} / 3 \mathrm{~N}\right)\right]^{-1}$

In the following steps, during our derivation let us use the general notation $\gamma_{\mathrm{c}}$ (where $\mathrm{c}=0$ for free of jamming, and $\mathrm{c}=1$ when PBJ effect occurs). Now we can develop as in the following steps.

We denote.$P_{e}(K)$ as the average error probability, the upper bound of the average bit error probability is given as [5] :

$\mathrm{P}_{\mathrm{e}}(\mathrm{K}) \leq \sum_{k_{f}=0}^{K-1} \quad \mathrm{P}_{\mathrm{f}}\left(\mathrm{k}_{\mathrm{f}}\right) \mathrm{P}_{\mathrm{ec}}\left(\mathrm{k}_{\mathrm{f}}\right)$

Where $\mathrm{K}$ is the total active users in the system, and $\mathrm{P}_{\mathrm{f}}\left(\mathrm{k}_{\mathrm{f}}\right)$ is the distribution of the full hits given as [5]:

$\mathrm{P}_{\mathrm{f}}\left(\mathrm{k}_{\mathrm{f}}\right)=\left(\begin{array}{l}(K-1) L \\ K_{f}\end{array}\right) \mathrm{P}_{\mathrm{f}}^{\mathrm{kf}}\left(1-\mathrm{P}_{\mathrm{f}}\right)_{\mathrm{f}}^{(\mathrm{K}-1-\mathrm{K}) \mathrm{L}}$

The term $\mathrm{P}_{\mathrm{ec}}\left(\mathrm{k}_{\mathrm{f}}\right)$ is the conditional bit error probability and it is given as:

$$
\mathrm{P}_{\mathrm{ec}}\left(\mathrm{k}_{\mathrm{f}}\right)=0.5 \mathrm{E}\left\{\exp \left(-0.5 \gamma_{\mathrm{c}} \mathrm{A}_{i, l}^{2}\right)\right.
$$

Since $\mathrm{A}_{\mathrm{i}, l}$ is distributed statistically as non-central chi-square random variable, its characteristic function is derived as [2] :

$$
\mathrm{E}\left\{\exp \left(-0.5 \gamma_{\mathrm{c}} \mathrm{A}_{i, l}^{2}\right)=1 /\left(1+\gamma_{\mathrm{c}} \sigma_{\varepsilon}^{2}\right) \exp \left[-\gamma_{\mathrm{c}} \mathrm{m}_{\varepsilon}^{2} /\left(2+2 \gamma_{\mathrm{c}} \sigma_{\varepsilon}^{2}\right)\right]\right.
$$

where $\mathrm{m}_{\varepsilon}$ is the mean of $\mathrm{A}_{i, l}^{2}$ and it can be expressed as [5]: $\mathrm{m}_{\varepsilon}=\varepsilon /(1+\varepsilon)$. And the variance of $\mathrm{A}_{i, l}^{2}$ is expressed as [5] $\sigma_{\varepsilon}^{2}=1 /(2+2 \varepsilon)$. The symbol $\varepsilon$ is tha fading factor. Then the expression of the upper bound on the average BER is given by substituting (9), (10), (11) and their related terms into (8) then we get: 
$\mathrm{P}_{\mathrm{e} 0}(\mathrm{~K}) \leq \sum_{K_{f}=0}^{K-1}\left(\begin{array}{l}(K-1) L \\ K_{f}\end{array}\right) 0.5 \mathrm{P}_{\mathrm{f}}^{\mathrm{kf}}\left(1-\mathrm{P}_{\mathrm{f}}\right)_{\mathrm{f}}^{(\mathrm{K}-1-\mathrm{K}) \mathrm{L}}\left(1+\gamma_{0} \sigma_{\varepsilon}^{2}\right)^{-1} \exp \left[-\gamma_{0} \mathrm{~m}_{\varepsilon}^{2} /\left(2+2 \gamma_{0} \sigma_{\varepsilon}^{2}\right)\right]$

For the case without jamming, and we get:

$\mathrm{P}_{\mathrm{e} 1}(\mathrm{~K}) \leq \sum_{K_{f}=0}^{K-1}\left(\begin{array}{l}(K-1) L \\ K_{f}\end{array}\right) 0.5 \mathrm{P}_{\mathrm{f}}^{\mathrm{kf}}\left(1-\mathrm{P}_{\mathrm{f}}\right)_{\mathrm{f}}^{(\mathrm{K}-1-\mathrm{K}) \mathrm{L}}\left(1+\gamma_{1} \sigma_{\varepsilon}^{2}\right)^{-1} \exp \left[-\gamma_{1} \mathrm{~m}_{\varepsilon}^{2} /\left(2+2 \gamma_{1} \sigma_{\varepsilon}^{2}\right)\right]$

For the case with jamming effect.

Finally, as a conclusion, the over all BER for the system under PBJ effect is

$\mathrm{P}_{\mathrm{e}}(\mathrm{K})=\rho \mathrm{P}_{\mathrm{e} 1}(\mathrm{~K})+(1-\rho) \mathrm{P}_{\mathrm{e} 0}$

Substituting with (12) and (13) into (14), then we get the final closed form expression of the BER for the hybrid SFH/DS SS, under the effect of PBJ can be expressed as:

$$
\begin{aligned}
& \mathrm{P}_{\mathrm{e}}(\mathrm{K})=\rho\left\{\sum_{K_{f}=0}^{K-1}\left(\begin{array}{l}
(K-1) L \\
K_{f}
\end{array}\right) 0.5 \mathrm{P}_{\mathrm{f}}^{\mathrm{kf}}\left(1-\mathrm{P}_{\mathrm{f}}\right)_{\mathrm{f}}^{\left(\mathrm{K}-1-\mathrm{K}_{\mathrm{f}} \mathrm{L}\right.}\left(1+\gamma_{1} \sigma_{\varepsilon}^{2}\right)^{-1} \exp \left[-\gamma_{1} \mathrm{~m}_{\varepsilon}^{2} /\left(2+2 \gamma_{1} \sigma_{\varepsilon}^{2}\right)\right]\right\} \\
& +(1-\rho)\left\{\sum_{K_{f}=0}^{K-1}\left(\begin{array}{l}
(K-1) L \\
K_{f}
\end{array}\right) 0.5 \mathrm{P}_{\mathrm{f}}^{\mathrm{kf}}\left(1-\mathrm{P}_{\mathrm{f}}\right)_{\mathrm{f}}^{(\mathrm{K}-1-\mathrm{K}) \mathrm{L}}\left(1+\gamma_{0} \sigma_{\varepsilon}^{2}\right)^{-1} \exp \left[-\gamma_{0} \mathrm{~m}_{\varepsilon}^{2} /\left(2+2 \gamma_{0} \sigma_{\varepsilon}^{2}\right)\right]\right\}
\end{aligned}
$$

the declaration of the various parameters for the closed form expression are:

- $\varepsilon$ is the system fading factor that depends on the channel type. where $\varepsilon=0$ for Rayleigh fading channel, while $\varepsilon=\infty$ for the AWGN channel, and $0<\varepsilon<\infty$ for Rician fading channel .

- $\mathrm{SNR}=\mathrm{E}_{\mathrm{b}} / \mathrm{N}_{\mathrm{o}}$ is the average signal to noise ratio.

- $\quad \mathrm{SJR}=\mathrm{E}_{\mathrm{b}} / \mathrm{N}_{\mathrm{j}}$ is the signal to jamming ratio.

- $\mathrm{L}$ is the number of the fading path.

- $P_{f}$ is the full hit probability (frequency full hit) $P_{f}=\left(1-2 N_{b}\right) q^{-1}$.

- $\mathrm{q}$ is the number of hopping frequencies.

\section{IV- NUMERICAL RESULTS}

Now we develop to use a MATHCAD programs to satisfy the BER performance for the system under consideration . the calculations are achieved by plotting the probability of error BER against SNR, and assuming the different system parameters. The number of frequency hop $q=10$, the number of active users is assumed to be $\mathrm{K}=10$, number of frequency hopping which is equal to, and the number of multipath $\mathrm{L}=4$. The different channel conditions can be controlled by changing the value of the 
fading factor $\varepsilon$. The fading factor is vary from $\varepsilon=\infty \mathrm{dB}$ for the AWGN channel conditions, while $\varepsilon=\infty$ $\mathrm{dB}$ for the Rayleigh fading channel conditions, and $\varepsilon=10 \mathrm{~dB}$ for Rician multipath fading channel.

In Fig.3 shows the BER performance of hybrid SFH/DS SSMA over AWGN channel under the effect of PBJ with different values of the jammer to signal ratio JSR. It is ranged from JSR=1, 2, 8, 10, and $20 \mathrm{~dB}$. It is noted that the increase of JSR, which means the increase of the jamming ratio more than the signal part, this leads to the more the system performance degradation.

Fig. 4 presents the BER performance of hybrid SFH/DS SSMA over Rayleigh fading channel under PBJ effect, and fixing SNR=20 dB. Now we can calculate the BER performance of a hybrid SFH/DS SSMA against signal to jamming ratio SJR. The total processing gain is fixed at 1000 i.e $=30 \mathrm{~dB}$. The effect of the fractional ratio on the system performance is shown in the plot by using different values of fraction jamming ratio ranged from $\rho=0.01,0.1,0.5,0.9$, and worst case of jamming $\rho=1$. it is noted from the plot that the increase of the fractional jamming part introduces the increase of the BER.

Fig.5 illustrate the BER performance of hybrid SFH/DS over Rician fading channel under PBJ effect, given that $\mathrm{JSR}=10 \mathrm{~dB}$, and with different value of processing gain, or more specifically with different values of the DS spread factor $\mathrm{N}=1,10$, and 20. It is noted that the system performance is enhanced with the increase of the overall system processing gain by increasing $\mathrm{N}$.

\section{V- CNCLUSION}

We have been presented throughout this paper, the BER performance analysis of the hybrid SFH/DS SSMA operating through AWGN channel, Rayleigh fading channel, and Rician fading channel. Moreover, such hybrid system is subjected to the effect of partial band noise or jamming. The performance is discussed assuming noncoherent reception model. A closed form expression for the hybrid system BER is derived for the system under consideration. The BER against SNR is taken as a performance measure. As well as the effect of increasing the jamming to signal ration JSR is discussed and system processing gain is discussed.

It is noted that the increase of JSR, which means the increase of the jamming ratio more than the signal part, this leads to the more the system performance degradation.

Moreover, it is noted that the increase of the fractional jamming part introduces the increase of the BER. Also, the system performance is enhanced with the increase of the overall system processing gain by increasing $\mathrm{N}$. 


\section{$\underline{\text { References }}$}

[1]Chanbum Park and Jea Hong Lee," Probability of error for hybrid DS/SFH spread spectrum over rician fading chaanel in the presence of multiple tone jamming", ISSSTA, 1992 [2] John G. Proakis ," Digital Communications", McGraw -Hill, 2000, fourth edition.

[ 3] R. Parsad, "CDMA for wireless personal communications", Artech house INC,1996.

[4] Bruce F. McGuffin "Jammed FH-FSK performance in Rayleigh and Nakagami fading" IEEE 2003 [5] Zhenhui Tan," Bounds on Performance of RS Coded Hybrid DS-SFH Spread Spectrum Multiple Access for Indoor Wireless Communications", International Conference on Communication Technology ICCT'93. 


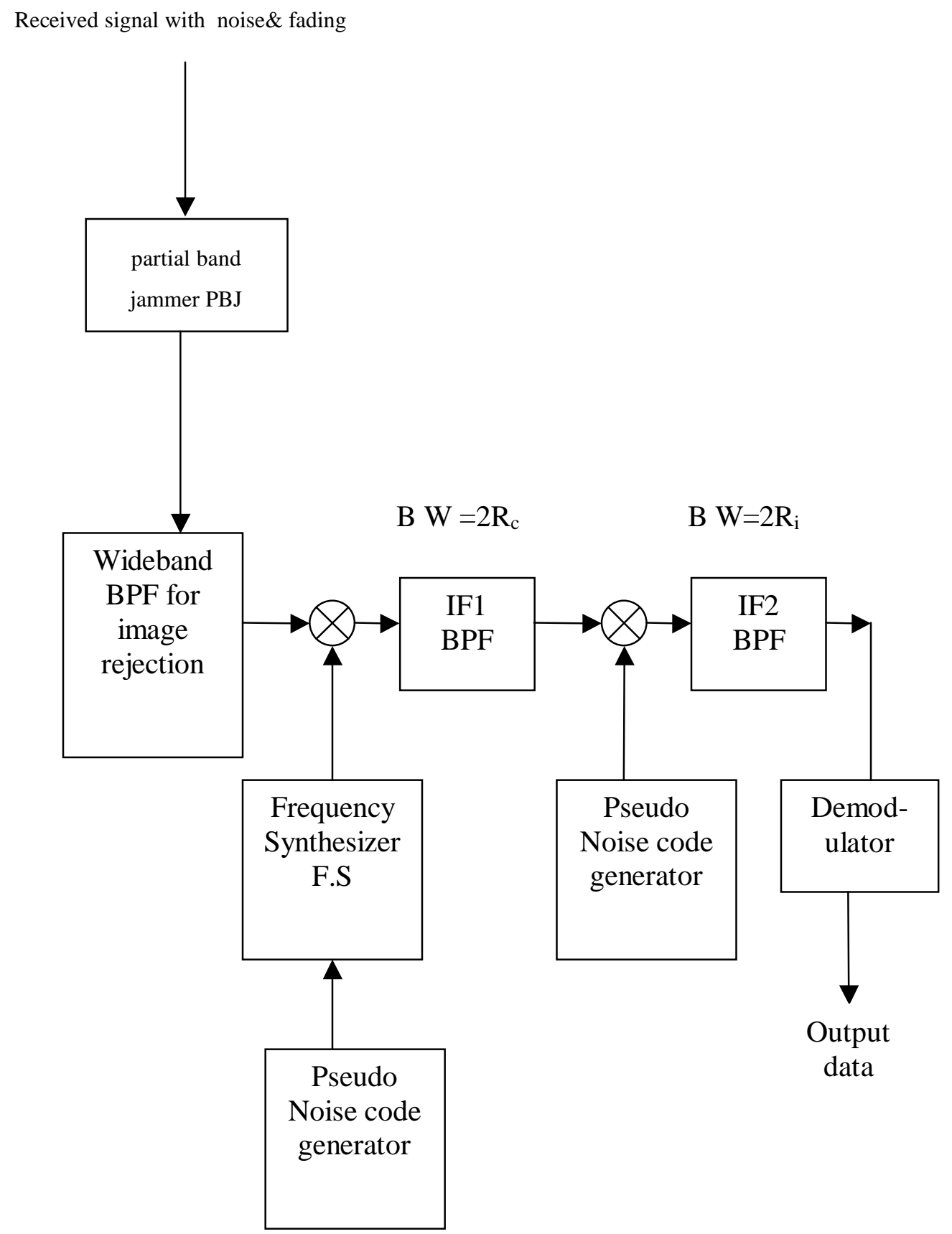

Figure 1 Receiver model for hybrid SFH/DS SSMA under PBJ effect 


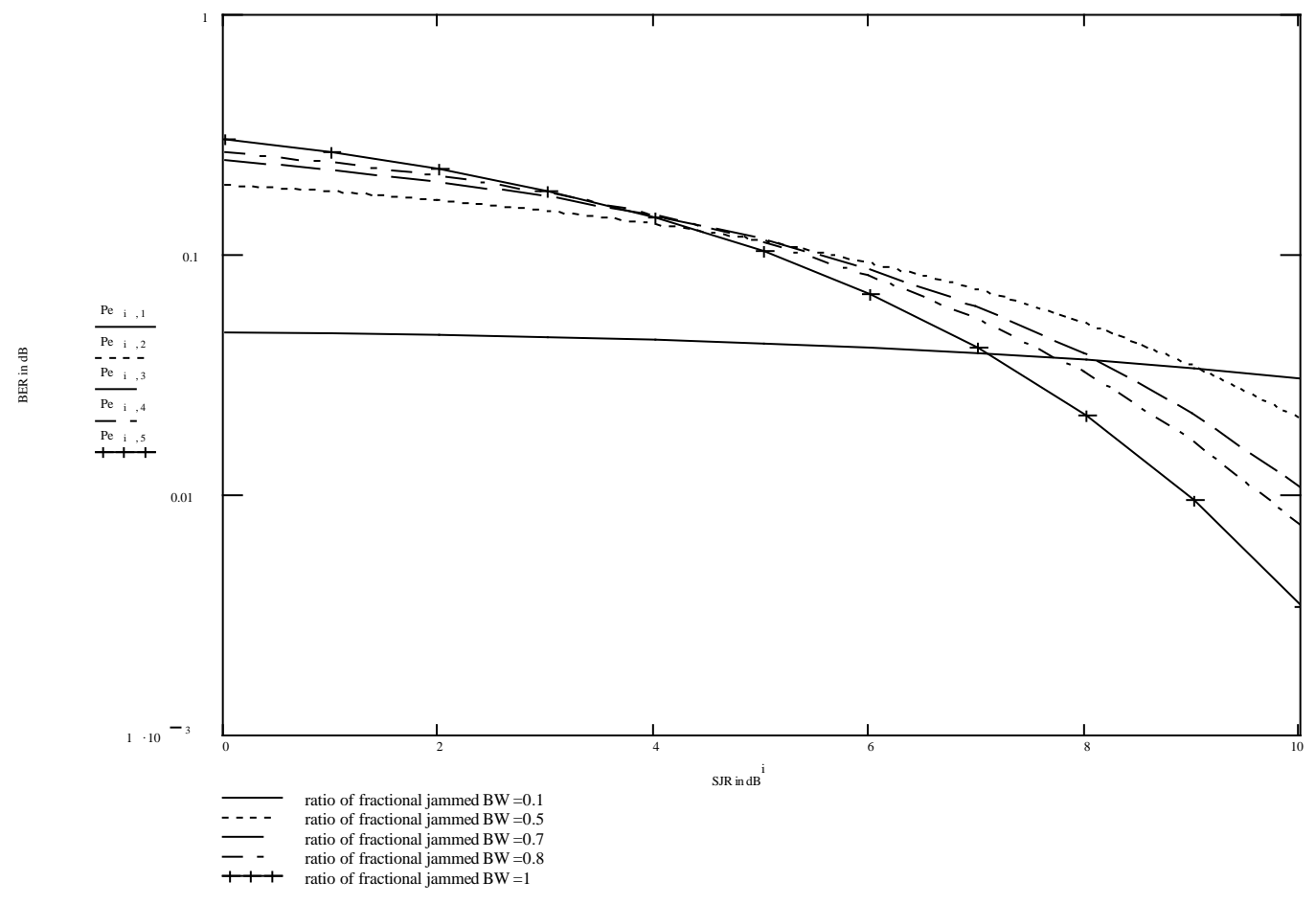

Figure 2 BER performance against signal to jamming ratio for the partial band jamming with different values of the fractional jammed ratio of the system BW.

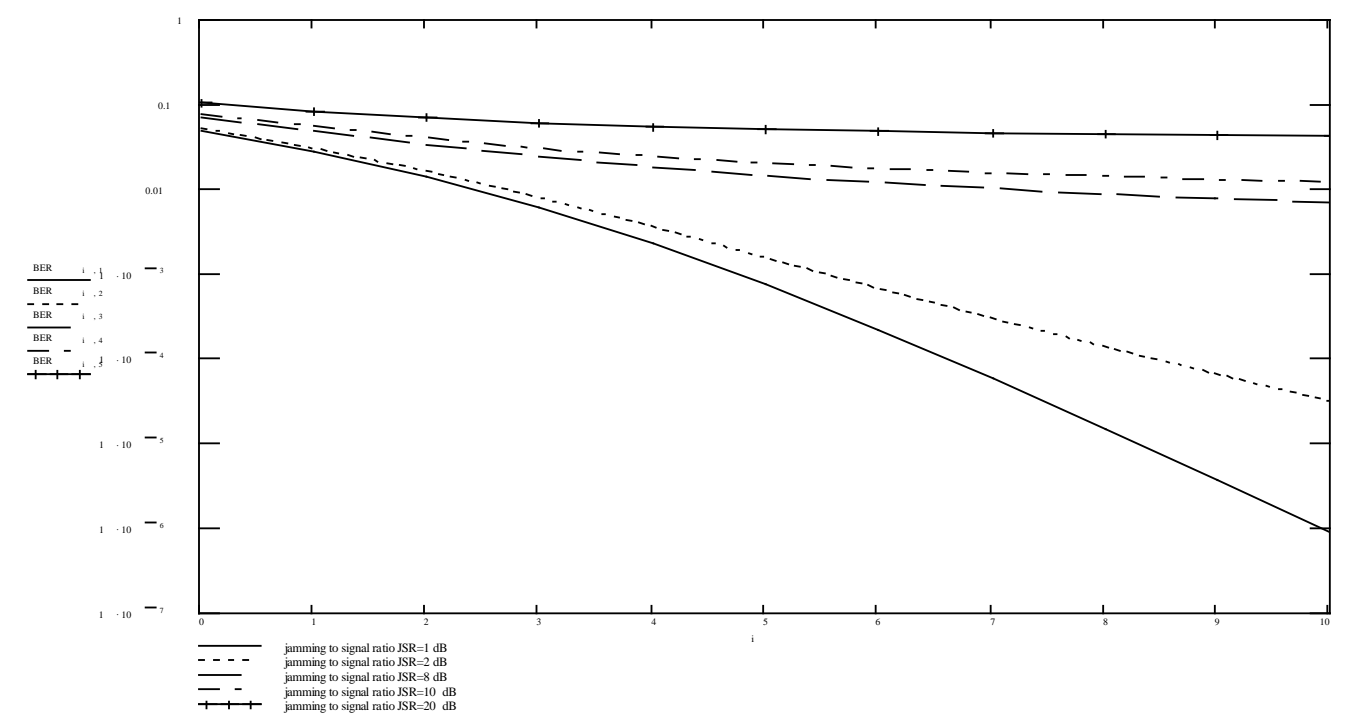

Figure 3 BER performance of hybrid SFH/DS SSMA over AWGN channel under the effect of PBJ with different values of the jammer to signal ration JSR. 


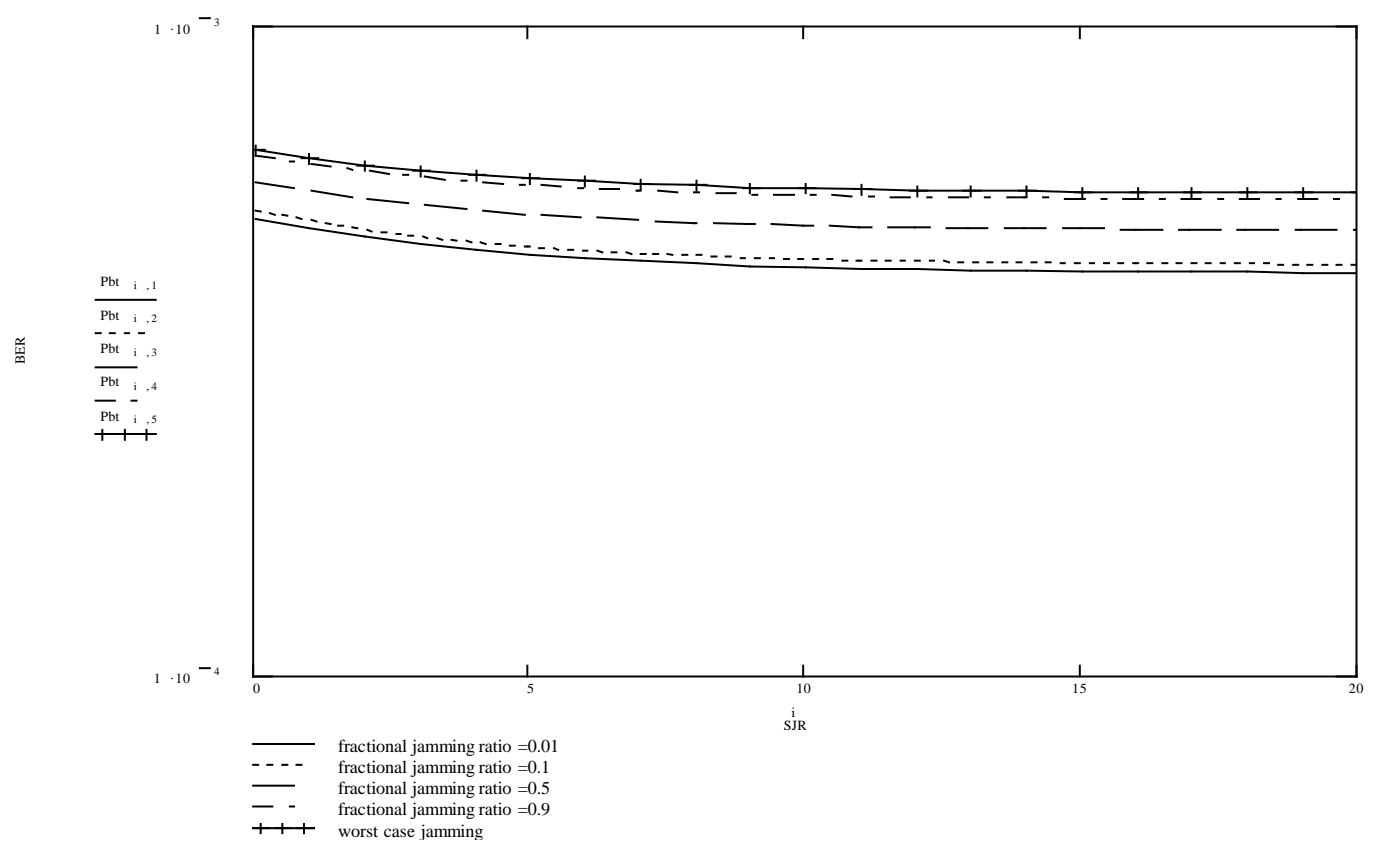

Fig. 4 BER performance of hybrid SFH/DS SSMA over Rayleigh fading channel under PBJ effect, $\mathrm{SNR}=20 \mathrm{~dB}$, different values of fraction jamming ratio.

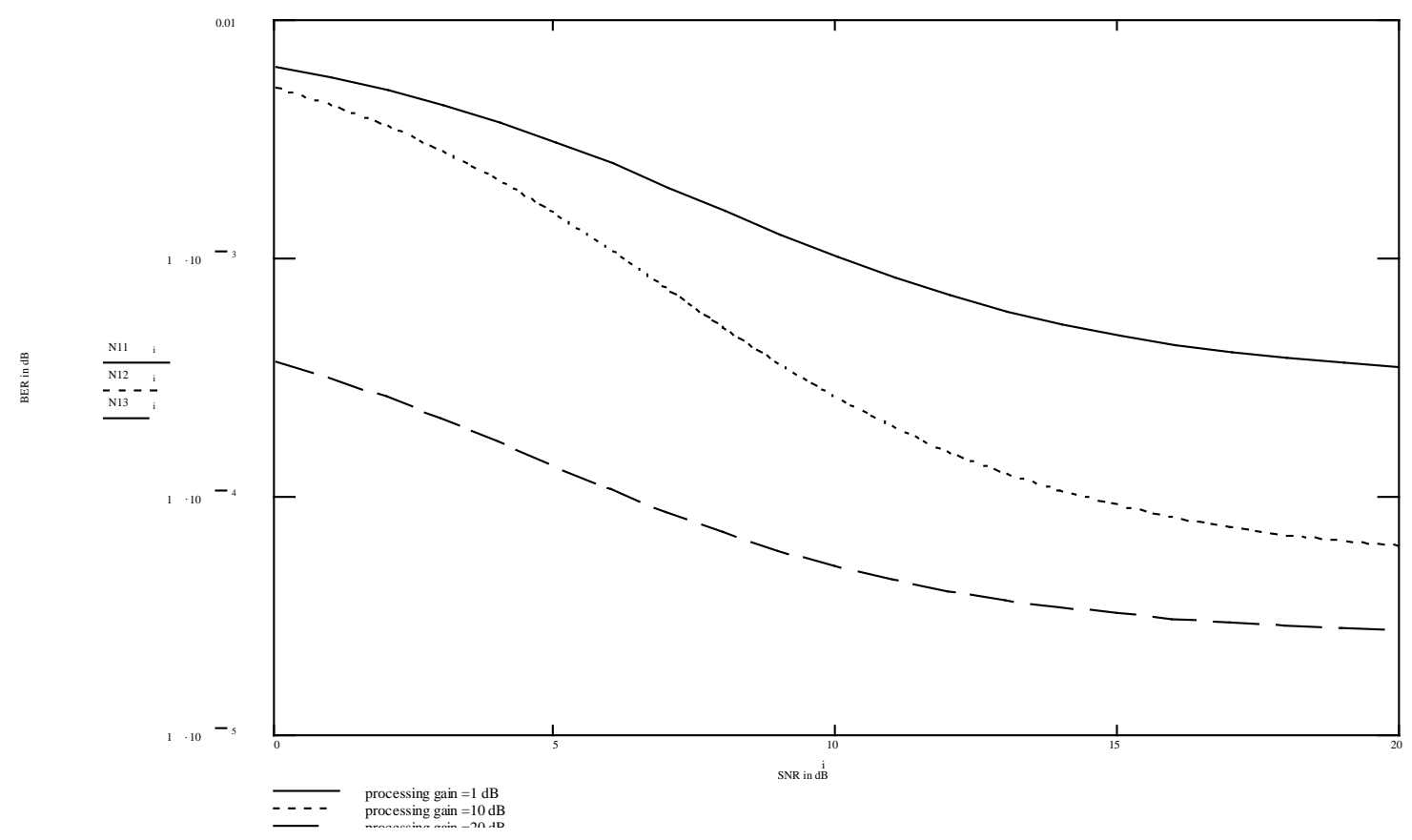

Figure 5 BER performance of hybrid SFH/DS over Rician fading channel under PBJ effect, given that $\mathrm{JSR}=10 \mathrm{~dB}$, and with different value of processing gain, 